\title{
Clinical Pharmacology
}

Open Access

ORIGINAL RESEARCH

\section{Antimalarial Drug Prescription: Evaluation of the Healthcare Professionals based on the Malian National Malaria Control Program (NMCP) Guidelines}

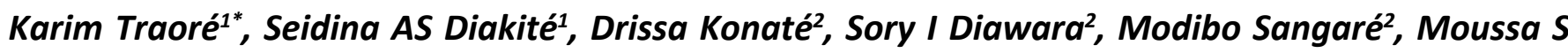 \\ Maiga $^{3}$, Fatoumata Daou ${ }^{1}$, Boubacar Fomba ${ }^{1}$, Mahamadou Diakité $^{1}$ and Sekou Bah ${ }^{1}$
}

${ }^{1}$ Faculty of Pharmacy of Bamako (FAPH), USTTB, Mali

${ }^{2}$ Faculty of Medicine and Odontostomatology of Bamako (FMOS), USTTB Mali

${ }^{3}$ Community Health Center of Kangaba, Mali

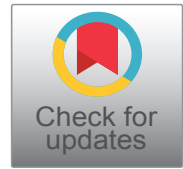

*Corresponding author: Karim Traoré, Faculty of Pharmacy of Bamako, Mali-ICER, MRTC, USTTB, BP 1805, Bamako, Mali

\begin{abstract}
Summary
Introduction: Since 2005, an artemisinin-based combination therapy has been recommended by the WHO and implemented by the National Malaria Control Program in Mali. The success of such strategy depends on the commitment of all stakeholders (patients and healthcare professionals). Therefore, it is necessary to assess the correct application of this recommendation.
\end{abstract}

Methods: We carried out the analysis of the prescription of antimalarial molecules based on the NMCP's guidelines in Kangaba and Bougouni Health Centers during the 2019 malaria transmission season from July $1^{\text {st }}$ to December $31^{\text {st }}$.

Results: The proportion of prescription with non-compliant antimalarial molecules was $67.1 \%$ and $53.3 \%$ in Kangaba and Bougouni, respectively. Up to $10.1 \%$ of the prescriptions in Kangaba and $26.2 \%$ in Bougouni were not conform to the NMCP dosage requirement. Treatment duration was not conform in $2.4 \%$ of prescriptions in Bougouni. The rapid diagnostic test was the mostly used biological test with $60.8 \%$ in Bougouni and $43.4 \%$ in Kangaba.

Conclusion: A large proportion of antimalarial prescriptions were not conform to the NMCP guidelines at our study sites in Mali.

\section{Keywords}

Prescription, Uncomplicated malaria, NMCP, Mali

\section{List of Abbreviations}

AL: Artemether-Lumefantrine; ASAQ: Artesunate-Amodiaquine; ACT: Artemisinin-based Combination Therapy;
EDSMV: Mali Demographic and Health Survey, volume V; MRTC: Malaria Research and Training Centre; NMCP: National Malaria Control Program; WHO: World Health Organization; ICER: International Center for Excellence in Research; USTTB: University of Sciences, Technics and Technologies of Bamako; RDT: Rapid Diagnostic Test

\section{Introduction}

The World Health Organization (WHO) estimates worldwide were 228 million cases of malaria and 405,000 deaths in 2018 as compared to 219 million cases including 435,000 deaths in 2017. Most malaria cases occur in sub-Saharan Africa in children under fiveyears-old and pregnant women [1]. In Mali, malaria remains a major endemic and leading cause of morbidity and mortality in health centers [2]. Despite the decreasing burden of malaria in our country through extensive success of the National Malaria Eradication Program (NMEP), the national health statistics show that malaria is still rang first among all diseases; It represents $39 \%$ of all outpatient visits in the health center and affects $19 \%$ of children under five-years-old [2].

Chloroquine (CQ) was massively produced in 1945. It provided a low toxicity and it was the least expensive and the most effective treatment of malaria. It was used to treat uncomplicated malaria or in the chemoprophylaxis. Chemoprophylaxis was one of the two pil-

Citation: Traoré K, Diakité SAS, Konaté D, Diawara SI, Sangaré M, et al. (2021) Antimalarial Drug Prescription: Evaluation of the Healthcare Professionals based on the Malian National Malaria Control Program (NMCP) Guidelines. Int Arch Clin Pharmacol 7:025. doi.org/10.23937/2572-3987.1510025 Accepted: February 03, 2021: Published: February 05, 2021

Copyright: (C) 2021 Traoré K, et al. This is an open-access article distributed under the terms of the Creative Commons Attribution License, which permits unrestricted use, distribution, and reproduction in any medium, provided the original author and source are credited. 
lars of the malaria eradication campaign in the 1950s and in the 1970s [3]. The first cases of chloroquine-resistance were reported in the world between 1957 and 1960 [4-7].

The resistance to antimalarial drugs is a serious hurdle for malaria control in endemic countries [8]. P. falciparum developed resistance to low-cost and well-tolerated antimalarial drugs such as chloroquine, amodiaquine (AQ), antifolates, and mefloquine (MQ) [3-6]. This has led the WHO and the National Malaria Control Programs (NMCP) to recommend the artemisinin-based combination therapy (ACT) for malaria treatment [9]. Nowadays, first signs of the emergence of parasites resistant to artemisinin derivatives drug have been reported in Southeast Asia $[10,11]$, but in Africa where ACTs are still very effective $[12,13]$. However, sub-Saharan Africa remains under threat due to the widespread use of ACTs along the increasing intercontinental human migrations. In addition, the circulation of sub-standard or counterfeit antimalarial drugs coupled to the non-adherence of patients to treatment may contribute to rapid selection of drug resistant malaria parasites. In 2006, the Malian NMCP adopted the artemisinin-based combination therapy (ACT) as the first-line treatment for uncomplicated malaria. In Mali, artemether-lumefantrine ( $\mathrm{AL}$ ) and amodiaquine-artesunate (AQ-AS) are used to treat uncomplicated malaria and the artesunate, artemether or quinine are used to treat severe malaria cases by intra-venous route.

The Malian NMCP has set up a strategic plan and developed guidelines with a protocol for the management of uncompleted and severe malaria cases [14]. The prescriber's responsibility is to institute correctly an early antimalarial drug treatment for confirmed malaria cases. Treatment should be in respect with the current standards and guidelines to reduce morbidity. The success of these recommendations in the new treatment policy will depend on the adherence and compliance of healthcare professionals and their patients [15]. No data were available on this protocol and its perception by prescribers. Consequently, we conducted this study to assess the conformity to the standards and guidelines of the NMCP of prescribed antimalarial drugs in Mali.

\section{Methods}

Our study took place in the health centers of Kangaba and Bougouni, two malaria endemic areas. Kangaba is located $80 \mathrm{~km}$ southwest of Bamako where the population is predominantly Malinké, while Bougouni is located $120 \mathrm{~km}$ south of Bamako where the population is predominantly Bambara and Senoufo. We conducted a cross-sectional study during malaria transmission season from July to December 2019. The sampling consisted of all prescriptions with at least one antimalarial drug and recorded in a pharmacy registry. The nature of the antimalarial drugs, its dosage, the duration of treatment and the type of prescribed antimalarial combination were analyzed based on the NMCP guidelines [14]. A non-compliant prescription was defined as any breach of one or more of the parameters listed above with respect to the NMCP guidelines. In the registries, we also collected information about biological diagnosis and the socio-demographic characteristics (age and sex of the patient). In addition, a report form was administered to all prescribers Data focusing on their professional qualification and their level of knowledge of the malaria treatment protocol.

Table 1: Presentation, dosage and duration of treatment for uncomplicated malaria with artemether 20 mg-lumefantrine 120 mg tablets.

\begin{tabular}{|c|c|c|c|c|c|c|}
\hline \multirow{2}{*}{ Age/weight } & \multicolumn{2}{|l|}{ Day 1} & \multicolumn{2}{|l|}{ Day 2} & \multicolumn{2}{|l|}{ Day 3} \\
\hline & Morning & Evening & Morning & Evening & Morning & Evening \\
\hline $05-14 \mathrm{Kg}$ (2 months to 3-years-old) & 1 tablet & 1 tablet & 1 tablet & 1 tablet & 1 tablet & 1 tablet \\
\hline $15-24 \mathrm{Kg}$ (4 to 6-years-old) & 2 tablets & 2 tablets & 2 tablets & 2 tablets & 2 tablets & 2 tablets \\
\hline $25-34 \mathrm{~kg}$ (7 to 13-years-old) & 3 tablets & 3 tablets & 3 tablets & 3 tablets & 3 tablets & 3 tablets \\
\hline$\geq 35 \mathrm{Kg}$ (14-years-old and more) & 4 tablets & 4 tablets & 4 tablets & 4 tablets & 4 tablets & 4 tablets \\
\hline
\end{tabular}

Table 2: Presentation, dosage and duration of treatment for uncomplicated malaria of artesunate-amodiaquine tablets.

\begin{tabular}{|l|l|l|l|l|}
\hline Age/weight & Presentation & Day 1 & Day2 & Day 3 \\
\hline $\begin{array}{l}\geq 4.5 \mathrm{~kg} \text { to }<9 \mathrm{~kg} \\
(2 \text { to } 11 \text {-months-old })\end{array}$ & $25 \mathrm{mg} / 67.5 \mathrm{mg}$ blister 3 tablets & 1 tablet & 1 tablet & 1 tablet \\
\hline $\begin{array}{l}\geq 9 \mathrm{~kg} \text { to }<18 \mathrm{~kg} \\
(1 \text { to } 11 \text {-years-old })\end{array}$ & $50 \mathrm{mg} / 135 \mathrm{mg}$ blister 3 tablets & 1 tablet & 1 tablet & 1 tablet \\
\hline $\begin{array}{l}\geq 18 \mathrm{~kg} \text { to }<36 \mathrm{~kg} \\
(6 \text { to } 13 \text {-years-old })\end{array}$ & $100 \mathrm{mg} / 270 \mathrm{mg}$ blister 3 tablets & 1 tablet & 1 tablet & 1 tablet \\
\hline $\begin{array}{l}\geq 36 \mathrm{~kg} \\
(\geq 14 \text {-years-old })\end{array}$ & $100 \mathrm{mg} / 270 \mathrm{mg}$ blister 6 tablets & 2 tablets & 2 tablets & 2 tablets \\
\hline
\end{tabular}


Types, dosages and duration of treatment combination prescribed by the NMCP are shown below (Table 1 and Table 2).

\section{Statistical analysis}

Data were collected on a report form, entered into Excel and analyzed using the statistical software Epi info 6.04.

\section{Results}

We recorded 300 prescriptions with at least one antimalarial drug (70\% in Kangaba and $30 \%$ in Bougouni) (Table 3). Non-compliant prescriptions represented $67.1 \%$ in Kangaba and 53.3\% in Bougouni. Dosages were non-compliant in Kangaba (10.1\%) and in Bougouni (26.2\%). All the prescriptions in Kangaba were fully compliant with the duration of treatment and only $2.4 \%$ in Bougouni were not. Among all the recommended ACTs, artemether-lumefantrine was the most prescribed with $100 \%$ in Kangaba and $97.6 \%$ in Bougouni (Table 4). Prescriptions were made when the diagnostic of malaria was confirmed by the rapid diagnostic test (RDT) and thick blood smear in Kangaba (83.3\%) and in Bougouni (87.8\%) (Table 3). The RDT was the mostly used biological test with $60.8 \%$ in Bougouni and $43.4 \%$ in Kangaba (Table 5). Forty-five (45) prescribers were registered, of which $66.7 \%$ in Kangaba and $33.3 \%$ in Bougouni with physicians $20 \%$ (9/45), nurses $57.8 \%(26 / 45)$ and midwives 22.2\% (10/45) (Table 3). Among the interviewees, $53 \%$ in Kangaba and $60 \%$ in Bougouni did not have sufficient knowledge of the existence of malaria treatment protocol according to the NMCP guidelines (Table 3).

\section{Discussion}

We conducted a cross-sectional study to evaluate the application of the national protocol for the management of uncomplicated malaria in Kangaba and Bougouni. This study took place from July to December 2019. We interviewed 45 healthcare professionals including nine (9) doctors, 26 nurses, 10 midwives and 300 children treated for malaria (210 cases in Kangaba

Table 3: Evaluation of antimalarial prescription at our two study sites.

\begin{tabular}{|c|c|c|c|c|c|}
\hline \multirow{2}{*}{\multicolumn{2}{|c|}{ Socio-demographic data of the prescribers }} & \multicolumn{2}{|c|}{ Kangaba } & \multicolumn{2}{|c|}{ Bougouni } \\
\hline & & \multirow{2}{*}{$\begin{array}{l}n \\
58\end{array}$} & \multirow{2}{*}{$\begin{array}{l}\text { \% } \\
27.6\end{array}$} & \multirow{2}{*}{$\begin{array}{l}\text { n } \\
27\end{array}$} & \multirow{2}{*}{\begin{tabular}{|l|}
$\%$ \\
30
\end{tabular}} \\
\hline \multirow{5}{*}{ Age ranges } & $0-5$ years-old & & & & \\
\hline & $6-8$ years-old & 12 & 5.7 & 3 & 3.3 \\
\hline & $9-13$ years-old & 10 & 4.8 & 5 & 5.6 \\
\hline & > 13-years-old & 130 & 61.9 & 55 & 61.1 \\
\hline & Total & 210 & 100 & 90 & 100 \\
\hline \multirow{2}{*}{ Gender } & Male & 99 & 47.1 & 39 & 43.3 \\
\hline & Female & 111 & 52.9 & 51 & 56.7 \\
\hline \multirow{4}{*}{ Qualification } & Physician & 5 & 16.7 & 4 & 26.7 \\
\hline & Nurse & 16 & 53.3 & 10 & 66.7 \\
\hline & Midewife & 9 & 30 & 1 & 6.6 \\
\hline & Total & 30 & 100 & 15 & 100 \\
\hline \multirow{3}{*}{ Awareness of the new protocol } & Yes & 14 & 47 & 6 & 40 \\
\hline & No & 16 & 53 & 9 & 60 \\
\hline & Total & & & & \\
\hline \multirow{3}{*}{$\begin{array}{l}\text { Malaria diagnostic confirmed by RDT and/or } \\
\text { thick blood smear }\end{array}$} & Yes & 175 & 83.3 & 79 & 87.8 \\
\hline & No & 35 & 16.7 & 11 & 12.2 \\
\hline & Total & & & & \\
\hline \multirow{4}{*}{$\begin{array}{l}\text { Antimalarial drugs for uncompleted malaria } \\
\text { treatment }\end{array}$} & Recommended ACT & 69 & 32.9 & 42 & 46.7 \\
\hline & Other ACTs & 0 & 0 & 4 & 4,4 \\
\hline & Monotherapy & 141 & 67.1 & 44 & 48.9 \\
\hline & Total & & & & \\
\hline \multirow{3}{*}{ Recommended dosage ACTs } & Conform & 62 & 89.9 & 31 & 73.8 \\
\hline & Non conform & 7 & 10.1 & 11 & 26.2 \\
\hline & Total & & & & \\
\hline \multirow{3}{*}{ Malaria treatment duration } & Conform & 69 & 100 & 40 & 95.2 \\
\hline & Non conform & 0 & 0 & 2 & 4.8 \\
\hline & Total & & & & \\
\hline
\end{tabular}


Table 4: Prescribed antimalarial drugs in uncomplicated malaria treatment per study sites.

\begin{tabular}{|l|l|l|l|}
\hline \multirow{2}{*}{ Prescribed antimalarial drugs } & \multicolumn{2}{|c|}{ Location $(\mathbf{n}=\mathbf{3 0 0}$} & \multirow{2}{*}{ Total $\mathbf{n}(\%)$} \\
\cline { 2 - 4 } & Bougouni $\mathrm{n}(\%)$ & Kangaba $\mathrm{n}(\%)$ & $110(36.7)$ \\
\hline Artemether lumfantrine (tablet) & $41(45.6)$ & $69(32.9)$ & $112(37.3)$ \\
\hline Artemether (injection) & $11(12.2)$ & $101(48.1)$ & $30(10)$ \\
\hline Quinine (tablet) & $13(14.4)$ & $17(8.1)$ & $24(8)$ \\
\hline Quinine (injection) & $13(14.4)$ & $11(5.2)$ & $14(4.7)$ \\
\hline Artemether (syrup) & $5(5.6)$ & $9(4.3)$ & $4(1.3)$ \\
\hline Artersunate (injection) & $1(1.1)$ & $3(1.4)$ & $2(0.7)$ \\
\hline Sulfadoxine-Pyrimethamine (tablet) & $2(2.2)$ & $0(0)$ & $1(0.3)$ \\
\hline Amodiaquine (syrup) & $1(1.1)$ & $0(0)$ & $1(0.3)$ \\
\hline Artesunate-Amodiaquine (tablet) & $1(1.1)$ & $0(0)$ & $2(0.7)$ \\
\hline Other ACTs & $2(2.2)$ & $0(0)$ & $\mathbf{3 0 0}(\mathbf{1 0 0})$ \\
\hline TOTAL & $\mathbf{9 ( 1 0 0 )}$ & $\mathbf{2 1 0}(\mathbf{1 0 0})$ & \\
\hline
\end{tabular}

Table 5: Distribution of patients according to type of biological tests.

\begin{tabular}{|l|l|l|l|}
\hline \multirow{2}{*}{ Biological testing } & \multicolumn{2}{|c|}{ Location $(\mathbf{n}=\mathbf{2 5 4})$} & \multirow{2}{*}{ Total $\mathbf{n}(\mathbf{\%})$} \\
\cline { 2 - 3 } & Bougouni $\mathrm{n}(\%)$ & Kangaba $\mathrm{n}(\%)$ & \\
\hline RDT & $48(60.8)$ & $76(43.4)$ & $124(48.8)$ \\
\hline Thick blood smear & $17(21.5)$ & $74(42.3)$ & $91(35.8)$ \\
\hline RDT + Thick blood smear & $14(17.7)$ & $25(14.3)$ & $39(15.4)$ \\
\hline TOTAL & $\mathbf{7 9 ( 1 0 0 )}$ & $\mathbf{1 7 5 ( 1 0 0 )}$ & $\mathbf{2 5 4}(\mathbf{1 0 0})$ \\
\hline
\end{tabular}

and 90 cases in Bougouni). This study provided the baseline information that can be used by the NMCP to plan its upcoming activities. Female patients were the most represented in Kangaba (52.9\%) and Bougouni (56.7\%). The most affected age group was patients aged 13 years old and older with in Kangaba (61.9\%) and in Bougouni (61.1\%) (Table 3). This result could be explained by the seasonal chemoprevention and the free management of malaria cases in children aged five years or younger, which would probably reduce the number of malaria episodes in this age group.

Prescribers were unaware of the national protocol for management malaria cases based on the NMCP in Bougouni (60\%) and in Kangaba (53\%). These proportions were higher as compared to about one third reported by Ahmed, et al. in Sudan in 2004 [16] and 15.5\% reported by Minzi in Tanzania in 2008 [17]. In Burkina Faso, LT. Ouédraogo, et al. in 2012 reported that prescribers affirmed to know the new treatment protocol for uncomplicated malaria in $84.6 \%$ [18]. This lack of knowledge of certain prescribers could be explained by the insufficiency or lack of training of health workers on the new national protocol. Indeed, the WHO and the Malian NMCP have recommended a biological confirmation of malaria cases using either the RDT or microscopy (thick blood smear) before any treatment is initiated. Most treated malaria cases were confirmed biologically in Bougouni (87.8\%) and Kangaba (83.3\%). In contrast, A. Soumana, et al. in 2016 in Niamey (46.5\%); M Meremikwu, et al. in 2007 in Nigeria (45\%) and T Diallo, et al. in 2017 (29\%) in Bamako reported a biological con- firmation of malaria before the treatment [19-21]. Confirmation by biology is one of Interventions to control malaria have been scaled up in recent years including the deployment RDTs to improve malaria surveillance and to guide malaria treatment in health facilities. The confirmation of parasite infection in a lab is important in the management of malaria and other febrile illnesses. It is necessary for clinicians to accurately know the actual infection status of each patient in order to manage properly febrile illnesses. As RDTs may be the only available tool for very remote areas, the issue of clinicians trusting the results is paramount if confirmation of parasite infection has to remain the basis for prescribing an anti-malarial drug.

Currently, ACT is the best choice to treat uncomplicated malaria cases. The efficacy and safety demonstrated of ASAQ and AL have led the WHO and the Malian NMCP to recommend these compounds for uncomplicated malaria treatment [22-24]. In this study, the recommended ACTs were prescribed only in $46.7 \%$ of cases in Bougouni and $32.9 \%$ in Kangaba. The prescription was not conform in 53.3\% in Bougouni and 67.1\% in Kangaba. A. Soumana, et al. in 2016 in Niamey reported that the antimalarial drugs prescription was not conform in $29.3 \%$ of patients [20]. Among the ACTs prescribed in Bougouni, $45.6 \%$ were AL combination versus $1.1 \%$ of ASAQ combination. All the ACTs prescribed in Kangaba were the artemether-lumefantrine combination. The non-prescription of amodiaquine-artesunate combination could be explained by the refusal of patients to take this drug due to the adverse effects of amodiaquine. 
If ACTs were used as the first-line treatment for uncomplicated malaria in Bougouni in $46.7 \%$, in contrast injectable Artemether was used in $48.1 \%$ in Kangaba. Rakotoarivelo R.A, et al. in 2012 in Madagascar reported that quinine was used as the first line treatment for uncomplicated malaria treatment instead of ACT [25]. Many reasons could explain this practice, such as vomiting or nausea or refusal to take the tablets (especially in children). These observations showed that the prescription of antimalarial drugs required a certain knowledge of the patient health and the healthcare setting. The use of injectable artemisinin in monotherapy for uncomplicated malaria treatment could be a factor for promoting the emergence and spread of resistance to artemisinin and its derivatives. In our study, $12.7 \%$ of patients were treated with an incorrect dosage of ACT. Patients were treated with ACTs with a non-compliant dosage in Bougouni (22\%) and (7.2\%) in Kangaba. Two patients had a Treatment duration was conform in all patients except two in Bougouni. Sow, et al. in 2016 in Guinea Conakry reported that $18.1 \%$ of patients received treatment for more than three (3) days and $39.8 \%$ received incorrect dosages [26].

Incomplete malaria treatment does not maintain an adequate antimalarial drug plasma level over a long enough time to eliminate all parasites in an individual. Thus, the selection of antimalarial drug-resistant parasites is promoted. The dosage was non-compliant for the management of uncomplicated malaria in Mali. This could be explained by the lack of training of agents on the national protocol and the lack of qualification of some prescribers. Hence, we emphasized the continuing training of prescribers at all levels of the health pyramid. This observation must challenge the NMCP and the Ministry of Health and Public Hygiene to implicate more the prescribers upstream for a large dissemination of the national guidelines for the management of malaria.

\section{Conclusion}

Our study showed a low utilization of the new protocol resulting in prescriptions that may be detrimental to patients. Proper use of ACTs is imperative to ensure their efficacy and to delay the emergence of drug resistance. We therefore suggest a large dissemination of the new malaria treatment protocol, and a continuing training on the the NMCP guidelines.

\section{Acknowledgements}

We thank the parents, guardians, and the children who participated in this study. We thank the technicians, clinicians, and the nursing staff for their assistance. We are grateful to many colleagues at the Malaria Research and Training Center (MRTC) for providing critical reviews of the manuscript.

\section{Ethics Approval}

Our study protocol was approved by the Ethics Com- mittee of the Faculty of Medicine and Pharmacy of the University of Sciences, Techniques and Technologies of Bamako (USTTB), Mali. We presented and explained our study to the administrative and customary authorities of the two localities before the start of our research activities.

\section{Consent for Publications}

All authors read and approved the final manuscript.

\section{Conflict of Interest}

Karim Traoré, Seidina AS Diakité, Drissa Konaté, Sory I. Diawara, Moussa S Maiga, Fatoumata Daou, Sékou Bah, Bboubacar Fomba and Mahamadou Diakité have no conflict of interest that are directly relevant to the content of this article.

\section{Funding}

This study is supported by a USTTB.

\section{References}

1. Rapport sur le paludisme dans le monde 2019. OMS: 232.

2. (2018) EDSM-VI, Cellule de Planification et de Statistiques (CPS/SSDSPF) Institut National de la Statistique (INSTAT) Centre d'Études et d'Information Statistiques (INFO-STAT) Bamako, Mali.

3. Packard RM (2014) The origins of antimalarial-drug resistance. N Engl J Med 371: 397-399.

4. Spencer HC (1985) Drug-resistant malaria--changing patterns mean difficult decisions. Trans R Soc Trop Med Hyg 79: $748-758$.

5. Wernsdorfer WH, D Payne (1991) The dynamics of drug resistance in Plasmodium falciparum. Pharmacol Ther 50: 95-121.

6. Wongsrichanalai C, Pickard Al, Wernsdorfer WH, Meshnick SR (2002) Epidemiology of drug-resistant malaria. Lancet Infect Dis 2: 209-218.

7. Rieckmann KH, DR Davis, DC Hutton (1989) Plasmodium vivax resistance to chloroquine? Lancet 2: 1183-1184.

8. WHO (2015) World malaria report 2015. WHO: Geneva.

9. (2011) Organization, $\mathrm{WH}$, Guidelines for the treatment of malaria. 2011, WHO.

10. Phyo AP, Nkhoma S, Stepniewska K, Ashley EA, Nair S, et al. (2012) Emergence of artemisinin-resistant malaria on the western border of Thailand: A longitudinal study. Lancet 379: 1960-1966.

11. Amaratunga C, Sreng S, Suon S, Phelps ES, Stepniewska K, et al. (2012) Artemisinin-resistant Plasmodium falciparum in Pursat province, western Cambodia: A parasite clearance rate study. Lancet Infect Dis 12: 851-858.

12. Ariey F WB, Amaratunga C, Beghain J, Langlois AC, Khim $\mathrm{N}$, et al. (2014) Un marqueur moléculaire du paludisme à Plasmodium falciparum rsistant à l'artémisinine. La nature 505: 50-55.

13. OMS (2011) Rapport mondial sur l'efficacité des médicaments antipaludiques et la pharmacorésistance: 2000-2010. Genève, S.O.m.d.I.s., Rapport mondial sur l'efficacité des médicaments antipaludiques et la pharmacorésistance: 20002010. . Genève, Suisse: Organisation mondiale de la santé. 
14. (2016) PNLP, Directives Nationales Pour La Prise En Charge Des Cas De Paludisme Au Mali.

15. Zurovac D, Rowe AK, Ochola SA, Noor AM, Midia B, et al. (2004) Predictors of the quality of health worker treatment practices for uncomplicated malaria at government health facilities in Kenya. Int J Epidemiol 33: 1080-1091.

16. Ahmed ME, Yousif (2004) Impact of the national protocol for malaria treatment on prescribing patterns in Gezira state, Sudan. East Mediterr Health J 10: 566-572.

17. Minzi OM, Haule AF (2008) Poor knowledge on new malaria treatment guidelines among drug dispensers in private pharmacies in Tanzania: The need for involving the private sector in policy preparations and implementation. East Afr J Public Health 5: 117-121.

18. Ouedraogo LT, KMD, Zongo PS (2012) Évaluation de l'application du protocole national de traitement des cas de paludisme simple en milieu rural au Burkina Faso. Santé Publique 353-363.

19. Meremikwu M, Okomo U, Nwachukwu C, Ita AO, EkeNjoku J, et al. (2007) Antimalarial drug prescribing practice in private and public health facilities in South-east Nigeria: A descriptive study. Malar J 6: 55.

20. A Soumana MK, Issofou B, Dima H, Daouda B, NDiaye O, Sall G (2016) Prescription d'antipaludiques dans le service de pédiatrie de l'Hôpital national de Lamordé, à Niamey. Bulletin de la Société de pathologie exotique.
21. T Diallo, Denou A, Coulibaly BF, Dakouo B, Coulibaly B (2017) Dispensation des antipaludiques dans les officines privées du district de Bamako, Mali. Antropo.

22. Schramm B, Valeh P, Baudin E, Mazinda CS, Smith R, et al. (2013) Efficacy of artesunate-amodiaquine and artemether-lumefantrine fixed-dose combinations for the treatment of uncomplicated Plasmodium falciparum malaria among children aged six to 59 months in Nimba County, Liberia: An open-label randomized non-inferiority trial. Malar J 12: 251.

23. Ngasala BE, Malmberg M, Carlsson AM, Ferreira PE, Petzold MG, et al. (2011) Efficacy and effectiveness of artemether-lumefantrine after initial and repeated treatment in children $<5$ years of age with acute uncomplicated Plasmodium falciparum malaria in rural Tanzania: A randomized trial. Clin Infect Dis 52: 873-882.

24. Getnet G, Fola AA, Alemu A, Getie S, Fuehrer H-P, et al. (2015) Therapeutic efficacy of artemether-lumefantrine for the treatment of uncomplicated Plasmodium falciparum malaria in Enfranze, north-west Ethiopia. Malar J 14: 258.

25. Rakotoarivelo RA, RC, Gottot S, Ravony Harintsoa L, Randrianiriana G, et al. (2012) Évaluation de la prise en charge des cas de fièvre et du paludisme par des médecins communautaires dans les Hautes Terres centrales de Madagascar 2009-2010.

26. Sow MS, Diallo MOS, Boushab BM, Savadogo M, Diawara IK (2016) Evaluation de l'utilisation du protocole national de prise en charge du paludisme simple des enfants à Matam, Guinée Conakry. Pan African Medical Journal. 